\title{
Correction to: Memory-based random walk for multi-query local community detection
}

\author{
Yuchen Bian ${ }^{1}$ (D) Dongsheng Luo ${ }^{1}$. Yaowei Yan ${ }^{1} \cdot$ Wei Cheng ${ }^{2} \cdot$ Wei Wang $^{3}$. \\ Xiang Zhang'
}

Published online: 11 October 2019

(c) Springer-Verlag London Ltd., part of Springer Nature 2019

\section{Correction to: Knowledge and Information Systems https://doi.org/10.1007/s10115-019-01398-3}

In the published article, Fig. 9a, b is the same figure. The correct Fig. 9a is given below.

The original article can be found online at https://doi.org/10.1007/s10115-019-01398-3.

Yuchen Bian

yub31@ist.psu.edu

Dongsheng Luo

du1262@ist.psu.edu

Yaowei Yan

yxy230@ist.psu.edu

Wei Cheng

weicheng@nec-labs.com

Wei Wang

weiwang@cs.ucla.edu

Xiang Zhang

xzhang@ist.psu.edu

1 College of Information Sciences and Technology, The Pennsylvania State University, University Park, PA 16802, USA

2 NEC Laboratories America, Princeton, USA

3 Department of Computer Science, University of California, Los Angeles, USA 


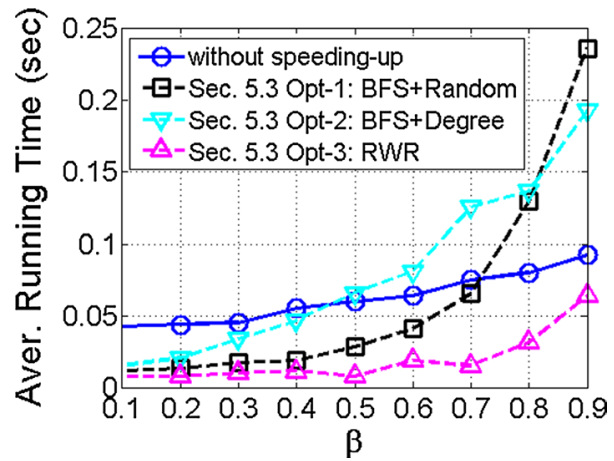

Publisher's Note Springer Nature remains neutral with regard to jurisdictional claims in published maps and institutional affiliations. 